Published in final edited form as:

Cancer Genet. 2016 September ; 209(9): 395-402. doi:10.1016/j.cancergen.2016.08.002.

\title{
Jumping translocations in myelodysplastic syndromes
}

\author{
Cecilia C. S. Yeunga,b, ${ }^{a}$, H. Joachim Deega ${ }^{a}$ Colin Pritchard ${ }^{c}$, David Wuc, and Min Fang ${ }^{a, b, c}$ \\ a Clinical Research Division, Fred Hutchinson Cancer Research Center, 1100 Fairview ave N, \\ Seattle, WA 98109, USA \\ b Department of Pathology, University of Washington, 1959 NE Pacific St, Seattle, WA 98195, \\ USA \\ c Department of Laboratory Medicine, University of Washington, 1959 NE Pacific St, Seattle, WA \\ 98195, USA
}

\begin{abstract}
Jumping translocations (JT) have been identified in numerous malignancies, including leukemia, but infrequently in patients with myelodysplastic syndromes (MDS). The responsible genetic region has been mapped to the JTB gene at 1q21, but breakpoints involving other chromosomal loci, such as $3 \mathrm{q}$ and 11q, have been described as well. We have characterized the pathological and mutational landscape, and the clinical course of 6 new MDS patients with jumping mutations using chromosome genomic array testing (CGAT) and target gene panel next generation sequencing. In addition, we have performed a literature review for other MDS cases with JTs as defined by ISCN 2013. Results support the concept that MDS in patients with jumping translocations has a poor prognosis with a high risk of progression to leukemia, and suggest that these patients warrant aggressive therapy, including HCT, early in the disease course.
\end{abstract}

\section{Keywords}

MDS; myelodysplasia; jumping translocation; CGAT; NGS

\section{Introduction}

Myelodysplastic syndromes (MDS) are a group of heterogeneous hematopoietic diseases with ineffective hematopoiesis and variable prognoses. Patients with high risk MDS may show rapid progression to acute leukemia, often refractory to therapy. Major determinants of disease progression in patients with MDS are included in the revised International Prognostic Scoring System (IPSS-R) (1-4). Allogeneic hematopoietic cell transplantation (HCT) offers potentially curative therapy. However, post-HCT disease recurrence is common, and only partially explained by currently scored risk factors. Outcome in these patients is poor $(5-8)$.

\footnotetext{
* Corresponding author. cyeung@FHCRC.org.
} 
Jumping translocations (JT) in neoplastic diseases are very rare cytogenetic phenomena where a segment of a particular chromosome is duplicated and inserted into several other chromosomes, resulting in multiple gains of this chromosomal segment via multiple translocations and possible loss of segments of the recipient chromosomes $(9,10)$. For the lay public, genetic counselors defined a jumping translocation ("Translocation Sauteuse") as describing a mitotic rearrangement whereby the same piece of one chromosome breaks off, on more than one occasion, and attaches to the tips of other chromosomes (11). Often, the site of breakage in the donor chromosome is characterized by the presence of an interstitial telomere, and this region offers the possibility of fusion with the recipient chromosomes. Although refined studies mapped the responsible locus to 1q11-21, and some reports specifically implicate the jumping translocation breakpoint (JTB) gene (12), JTs involving loci such as 3q, 11q, and other genomic loci have been described. However, the mutational landscape of these MDS cases with JT has not been defined previously. In the present study, we aimed at characterizing the mutational landscape by performing chromosomal genomic array testing (CGAT) and target gene panel next generation sequencing in MDS patients with JT seen at our center from 2000 to 2013. We posit that a JT represents a very high-risk cytogenetic abnormality, and propose that such patients should be considered for early aggressive therapy including HCT even if other disease parameters suggest low risk disease.

\section{Material and methods}

\section{Study population}

A retrospective review of the cytogenetic data of cases of MDS and MDS-based myeloid neoplasms observed over the past 10 years at the Seattle Cancer Care Alliance (SCCA) was conducted. Cases where conventional cytogenetic studies identified a JT during the disease course were selected for analysis.

All patients had been consented under standard SCCA patient consent for research studies. The studies were approved by the Fred Hutchinson Cancer Research Center institutional review board. Cytogenetics (including karyotype, fluorescence in situ hybridization (FISH), and CGAT), pathology and flow cytometry data were correlated with clinical course and outcomes.

\section{DNA extraction}

Sources of DNA for CGAT included fresh or frozen marrow and archived fixed cell pellets. DNA from fresh bone marrow and fresh frozen marrow aspirates was extracted using the Qiagen-PureGene method (Germantown, MD) according to the manufacturer's protocol. For DNA extraction from archived samples, cell pellets in methanol/acetic acid fixative were washed 3 times with cold phosphate-buffered saline (PBS), re-suspended in $100 \mu$ of PBS, and loaded onto the Qiagen EZ1 Advanced XL according to the Qiagen EZ1 Virus Mini Kit v2.0. Elution volume was $60 \mu$ l. Extraction was performed per manufacturer guidelines. DNA was stored at $4{ }^{\circ} \mathrm{C}$.

DNA quality was assessed by NanoDrop 2000 Spectrophotometer (Thermo Scientific, Waltham, MA), which measures DNA concentration and purity by $260 / 280 \mathrm{~nm}$ readings. 
The DNA was also visualized on a $1 \%$ agarose gel with ethidium bromide to detect/exclude degradation. The criteria for acceptable DNA quality included visible bands by $1 \%$ agarose gel and spectrophotometer reading by A260nm/A280nm ratio of 1.4-2.

\section{Chromosomal genomic array testing (CGAT)}

CGAT for the detection of DNA copy number aberration (CNA) or copy-neutral loss of heterozygosity $(\mathrm{cnLOH})$ by single nucleotide polymorphism (SNP) genotyping was performed with CytoScan HD (Affymetrix, Santa Clara, CA) according to the manufacturer's protocol. The size filter for an abnormal call was $100 \mathrm{~kb}$ (and 25 probes) for $\mathrm{CNA}$ and $10 \mathrm{Mb}$ for $\mathrm{cnLOH}$.

\section{Targeted gene panel next generation sequencing}

We preformed targeted sequencing of 194 cancer-related genes (Oncoplex, University of Washington, Seattle, WA) with 2 101-bp, paired-end reads and on a HiSeq2000 sequencing system or a MiSeq (Illumina, San Diego, CA). An average of $>850,000$ base pairs of DNA were sequenced at a depth of $>500 \times$ coverage, as described in detail before (13).

\section{Literature review}

In addition, a literature review was conducted in PubMed and Web of Science using the following terms:

((“Myelodysplastic Syndromes”[Mesh] AND Case Reports[ptyp])) AND “Jumping Translocations"

(“Myelodysplastic Syndromes”[Mesh]) AND jumping translocations

"Myelodysplastic Syndromes”[Mesh] AND “Jumping Translocations"

“Jumping Translocations” AND (Case Reports[ptyp])

Papers were reviewed and cases that did not comply with the ISCN (2013) definition of JT were excluded.

\section{Results}

We identified six JT-MDS cases. Four patients were male and 2 female, 53-76 years of age. Two patients presented with refractory anemia with excess blasts-2 (RAEB-2), one with RAEB-1, one with therapy-related MDS, one with unclassifiable MDS/MPN, and one with refractory cytopenia with unilineage dysplasia (RCUD) (14). One patient had both initial diagnostic and post-transplant relapse samples available for comparison. The mean time to JT identification from initial MDS diagnosis or, in transplanted patients, from the time of HCT, was 22 months. In 5 of the 6 cases, JT involved chromosome 1q, and in one case chromosome $3 \mathrm{q}$.

Five of the 6 patients received HCT (one patient was transplanted twice), and one patient died following MDS transformation to AML without being transplanted. At the time of HCT, MDS had progressed to AML in 4 of 5 patients. With 1-2 years of follow up, 3 [2 
after HCT and one from progressive disease without transplantation] of 6 patients have died from relapsed disease. Three patients are surviving, one of these in relapse.

The index case of this series (patient 1) was a 66-year-old male who initially presented with refractory anemia with excess blasts (5\%) with normal karyotype in 2011 (Figure 1). The blast count declined in response to Vidaza but in the fall of 2012, the blast count had increased again and cytogenetics showed $\mathrm{t}(\mathrm{X} ; 12)$ and multiple JTs involving chromosome 1q. CGAT showed multiple 1q gains, $\mathrm{cnLOH}$ of $4 \mathrm{q}$, and deletion of 18q. The region of $4 \mathrm{q}$ cnLOH involved TET2 - tet methylcytosine dioxygenase 2.

Targeted sequencing showed a novel single base pair mutation in TET2 (c.3594 + 5G $>$ A) at cDNA position 3594 resulting in a splice site mutation, confirmed by two separate informatics programs (SIFT and fruitfly.org). In addition, there was a missense mutation in $S F 3 B 1$ at codon 625 (c.1873C >T:p.R625C) $(15,16)$, and a $C B L$ single base pair mutation (c. 1477C>T:p.L493F) leading to an amino acid change. This mutation, not previously described in MDS, was confirmed by informatics prediction scores (pheophen, SIFT, Mutation Taster, and Gerp), implying a detrimental effect. Further, a single base pair mutation was present in SRSF2 (c.284C>A:p.P95H). This mutation, which leads to an amino acid change has been described in other MDS cases before $(15,17-19)$.

HCT was recommended. The patient was conditioned with fludarabine, and 2 Gy total body irradiation (TBI) and received a transplant from an HLA-A,B,C, DRB1 matched, DQB1 antigen mismatched unrelated donor. He remains well with donor cell engraftment and without evidence of relapse (normal cytogenetics) 2 years after transplantation.

Details for the clinical course and the pertinent cytogenetic progression time points for all 6 new JT-MDS cases are summarized in Table 1.

Comparative studies by karyotype, CGAT, and targeted NGS gene panel were carried out for the first four JT-MDS cases. In one patient whose disease relapsed post-HCT a similar analysis was carried out on the relapse sample. A complete analysis was not possible in patients 5 and 6 . The comparative results are detailed in Table 1. CGAT confirmed the complex nature of these JT cases, and in many cases showed additional abnormalities that were not seen by conventional cytogenetics, such as cnLOH. Recurrent mutations (by NGS) involved TET2, which in 1 case corresponded to $\mathrm{cnLOH}$ of $4 \mathrm{q}$ spanning the TET2 locus, 2 cases had 2 mutations each in the TET2 locus, and the fourth case had a splicing mutation of TET2.

\section{Literature review}

To assess current information on JTs in MDS more comprehensively, we reviewed 52 publications describing JTs in any malignancies, and focused on 12 papers, which included JTs in patients with AML, MDS, or MPN. We excluded 6 papers, which discussed segmental jumping translocations or amplification of the $M L L$ gene because they did not qualify under the ISCN definition of a jumping translocation.

Details of published cases are provided in Table 2. Hatakeyama et al. described a JT-MDS patient who subsequently developed AML (reportedly 10 years after initial diagnosis) (20). 
Najfeld, described 5 MDS patients with 1qJT in a letter, four of whom also experienced transforming to AML (23). We did not consider several cases from earlier publications by Najfeld et al. because they did not match the ISCN definition of jumping translocations based on the published karyotypes (24,25). More recently Lizcova et al. and McGrattan et al. described a wide range of clinical presentations in both older and very young patients as well as descriptions of JTs involving different chromosomal regions $(21,22)$.

\section{Discussion}

JTs have been described infrequently in patients with MDS. We identified 9 cases in the literature and described 6 new cases, bringing the total to 15 documented cases.

The data indicate that MDS patients with JTs often have a poor prognosis with high rates of disease progression or relapse and mortality. Among the 8 cases in the literature 7 had transformed to AML. The time to the acquisition of JT was 20 and 21 months, respectively, in the two papers, which reported this information. The interval in the 6 cases reported here (all diagnosed since 2008, ranged from 12 to 39 months, one patient received a HCT shortly after acquisition of JT but before AML transformation of MDS), and the interval refers to the time from transplantation to relapse. All 6 cases of JT-MDS from our institution were acquired with a range of 12-39 months between initial diagnoses of the patient's myeloid neoplasm or first HCT to when the JT was first documented. Five of our 6 cases of JT-MDS showed complex karyotype, making the very high-risk IPSS-R cytogenetic classification category. Very likely, the better diagnostic tools, classification schemes, and modern therapies, as well as the more widely adopted practice of HCT in patients with MDS also improved the overall clinical outcome. However, even with the apparent (minor) improvement, MDS with JTs appears to have a more aggressive clinical course than other patients.

Three of the 6 patients reported here died within 18 months of JT acquisition. One patient relapsed at 12 months post-HCT, and 2 patients (33\%) remain in clinical remission post allogenic transplantation at last contact (median follow-up 2 years). Previous reports from our center show 3 year progression-free survival (PFS) of 28\% and 44\% for MDS patients who have received HCT following nonmyeloblative and myeloablative conditioning, respectively (26). However, given the small number of cases with JTs, a meaningful statistical comparison was not possible.

As indicted, the literature on JTs in MDS is limited. The small case series by Najfeld et al. focused on 1qJT and reviewed the clinical characteristics with conventional cytogenetic studies including karyotyping and FISH. However, the $J T B$ gene has been mapped to a gene rich region on chromosome 1q21 (20), and has been named for its propensity to create partial duplications with unbalanced translocations. JTB's genomic structure contains five exons and 4 introns and spans over $3 \mathrm{~kb}(20)$. In this case report, the fusion point was located in intron 4, resulting in a truncated protein lacking the exon 5 trans-membrane and intracellular domains. We chose to exclude any previously described cases of JT, where the published data did not match the ISCN definition. We also disregarded papers describing segmental jumping translocations or segmental amplifications involving the $M L L$ gene, as 
we considered this to be more congruent with gene amplification aberration and not matching the criteria set forth by the ISCN.

We also attempted to determine the synergistic information that can be obtained from performing both CGAT and targeted NGS gene panel analysis. The data suggest that in MDS cases with $\mathrm{cnLOH}$, the underlying biology and mutational landscape are complex. While conventional cytogenetics is required for the diagnosis of JTs, CGAT can provide additional information. Importantly, CGAT has allowed a more detailed examination of the breakpoints for the JTs and have shown that there is minor variability to the exact breakpoints likely between the various clones even when this is not apparent by conventional cytogenetic karyotyping. Adding information obtained by NGS can identify sequence specific mutations in genes that are shown to have loss of heterozygosity by CGAT.

Four cases in our series had TET2 mutations and one of the four cases also showed cnLOH of the $4 \mathrm{q}$ region involving the TET2 gene by concurrent CGAT testing, resulting in homozygous expression of this mutation. Conceivably, the TET2 mutation indicates an unstable genomic state and, when present in association with JTs, portends a poorer prognosis. Somatic TET2 mutations have been described in approximately $5 \%$ of older individuals without hematologic malignancy (27). However, TET2 mutations have clearly been linked to myeloid dysfunction (28) and clonal disease (29), and, more importantly, responsiveness to hypomethylating agents such as azacitabine or decitabine (30).

SF3B1 mutations are the second most common mutation in MDS. We observed this mutation in 3 of 6 JT cases in this study. Recently, Malcovati et al. (31) suggested SF3B1 mutated MDS were not likely to develop detrimental subclonal mutations. However, our data suggest that there may be some caveats to this observation and in two of our cases there were concurrent TET2 mutations with $S F 3 B 1$ mutation. One of them, JT2, demonstrated loss of TET2 mutation but retention of the $S F 3 B 1$ mutation during relapse, when JT was acquired. It may be that the acquisition of JT trumps the beneficial effect of any preexisting or subsequently attained $S F 3 B 1$ mutations.

In summary, we identified 6 new cases of JTs in patients with MDS and reviewed another 9 cases reported previously. The data suggest that JTs are associated with disease progression to AML. Despite improved management including HCT, JT-MDS is associated with high relapse and mortality rates. HCT may be useful in prolonging survival and may prove curative. Additional research is required to further define the biology of JTs. It may be warranted to classify patients with JT-MDS into higher-risk category (similar to RAEB-2 or evolving AML).

\title{
Acknowledgments
}

\author{
1. SCCA cytogenetics laboratory. \\ 2. Douglas M Shane, Arnold Library. \\ 3. Genomics laboratory, UW Department of Laboratory Medicine. \\ Funding
}


CCSG grant $=$ National Cancer Institute, National Institutes of Health, Bethesda, MD (CA018029 and CA015704). This study was supported in part a Pilot \& Feasibility grant awarded to

M. Fang from DK056465 Core Center of Excellence in Hematology.

Disclosures

Dr. Yeung has received research funding from Gilead and Dr. Fang has received research funding from Affymetrix, both are on projects that are unrelated to the project.

\section{References}

1. Greenberg PL, Tuechler H, Schanz J, et al. Revised international prognostic scoring system for myelodysplastic syndromes. Blood. 2012; 120:2454-2465. [PubMed: 22740453]

2. Schanz J, Tuchler H, Sole F, et al. New comprehensive cytogenetic scoring system for primary myelodysplastic syndromes (MDS) and oligoblastic acute myeloid leukemia after MDS derived from an international database merge. J Clin Oncol. 2012; 30:820-829. [PubMed: 22331955]

3. Estey EH, Keating MJ, Dixon DO, et al. Karyotype is prognostically more important than the FAB system's distinction between myelodysplastic syndrome and acute myelogenous leukemia. Hematol Pathol. 1987; 1:203-208. [PubMed: 3504436]

4. Greenberg P, Cox C, LeBeau MM, et al. International scoring system for evaluating prognosis in myelodysplastic syndromes. Blood. 1997; 89:2079-2088. [PubMed: 9058730]

5. Deeg HJ, Scott BL, Fang M, et al. Five-group cytogenetic risk classification, monosomal karyotype, and outcome after hematopoietic cell transplantation for MDS or acute leukemia evolving from MDS. Blood. 2012; 120:1398-1408. [PubMed: 22767498]

6. Lim Z, Brand R, Martino R, et al. Allogeneic hematopoietic stem-cell transplantation for patients 50 years or older with myelodysplastic syndromes or secondary acute myeloid leukemia. J Clin Oncol. 2010; 28:405-411. [PubMed: 20008642]

7. Mielcarek M, Storer BE, Sandmaier BM, et al. Comparable outcomes after nonmyeloablative hematopoietic cell transplantation with unrelated and related donors. Biol Blood Marrow Transplant. 2007; 13:1499-1507. [PubMed: 18022580]

8. Yeung CC, Gerds AT, Fang M, et al. Relapse after allogeneic hematopoietic cell transplantation for myelodysplastic syndromes: analysis of late relapse using comparative karyotype and chromosome genome array testing. Biol Blood Marrow Transplant. 2015; 21:1565-1575. [PubMed: 25953732]

9. Berger R, Bernard OA. Jumping translocations. Genes Chromosomes Cancer. 2007; 46:717-723. [PubMed: 17444494]

10. McGowan-Jordan J, Shaffer LG, Schmid M, ISCN (2013). An International System for Human Cytogenetic Nomenclature (2013). Karger. 2013:140.

11. Gardner, RJM.; Sutherland, GR.; Shaffer, L. Chromosome Abnormalities and Genetic Counseling (Oxford Monographs on Medical Genetics). 4th. Oxford University Press; 2011.

12. Hatakeyama S, Osawa M, Omine M, et al. JTB: a novel membrane protein gene at 1q21 rearranged in a jumping translocation. Oncogene. 1999; 18:2085-2090. [PubMed: 10321732]

13. Pritchard CC, Salipante SJ, Koehler K, et al. Validation and implementation of targeted capture and sequencing for the detection of actionable mutation, copy number variation, and gene rearrangement in clinical cancer specimens. J Mol Diagn. 2014; 16:56-67. [PubMed: 24189654]

14. Swerdlow, SH.; Campo, E.; Harris, NL., et al. WHO Classification of Tumours of Haematopoietic and Lymphoid Tissues. 4th. IARC Press; Lyon, France: 2008.

15. Papaemmanuil E, Gerstung M, Malcovati L, et al. Clinical and biological implications of driver mutations in myelodysplastic syndromes. Blood. 2013; 122:3616-3627. quiz 3699. [PubMed: 24030381]

16. Visconte V, Makishima H, Jankowska A, et al. SF3B1, a splicing factor is frequently mutated in refractory anemia with ring sideroblasts. Leukemia. 2012; 26:542-545. [PubMed: 21886174]

17. Yoshida K, Sanada M, Shiraishi Y, et al. Frequent pathway mutations of splicing machinery in myelodysplasia. Nature. 2011; 478:64-69. [PubMed: 21909114] 
18. Xu L, Gu ZH, Li Y, et al. Genomic landscape of CD34+ hematopoietic cells in myelodysplastic syndrome and gene mutation profiles as prognostic markers. Proc Natl Acad Sci USA. 2014; 111:8589-8594. [PubMed: 24850867]

19. Menezes J, Acquadro F, Wiseman M, et al. Exome sequencing reveals novel and recurrent mutations with clinical impact in blastic plasmacytoid dendritic cell neoplasm. Leukemia. 2014; 28:823-829. [PubMed: 24072100]

20. Hatakeyama S, Fujita K, Mori H, et al. Shortened telomeres involved in a case with a jumping translocation at 1q21. Blood. 1998; 91:1514-1519. [PubMed: 9473214]

21. McGrattan P, Logan A, Humphreys M, et al. Jumping translocation in acute monocytic leukemia (M5b) with alternative breakpoint sites in the long arm of donor chromosome 3. Med Oncol. 2010; 27:667-672. [PubMed: 19629764]

22. Lizcova L, Zemanova Z, Malinova E, et al. Jumping translocations in bone marrow cells of pediatric patients with hematologic malignancies: a rare cytogenetic phenomenon. Cancer Genet. 2011; 204:348-349. [PubMed: 21763634]

23. Najfeld V, Tripodi J, Scalise A, et al. Jumping translocations of the long arms of chromosome 1 in myeloid malignancies is associated with a high risk of transformation to acute myeloid leukaemia. Br J Haematol. 2010; 151:288-291. [PubMed: 20738298]

24. Najfeld V, Hauschildt B, Scalise A, et al. Jumping translocations in leukemia. Leukemia. 1995; 9:634-639. [PubMed: 7723397]

25. Najfeld V, Chen A, Scalise A, et al. Myelodysplastic syndrome transforming to acute promyelocytic-like leukemia with trisomy and rearrangement of chromosome 11. Genes Chromosomes Cancer. 1994; 10:15-25. [PubMed: 7519869]

26. Scott BL, Sandmaier BM, Storer B, et al. Myeloablative vs nonmyeloablative allogeneic transplantation for patients with myelodysplastic syndrome or acute myelogenous leukemia with multilineage dysplasia: a retrospective analysis. Leukemia. 2006; 20:128-135. [PubMed: 16270037]

27. Busque L, Patel JP, Figueroa ME, et al. Recurrent somatic TET2 mutations in normal elderly individuals with clonal hematopoiesis. Nat Genet. 2012; 44:1179-1181. [PubMed: 23001125]

28. Scopim-Ribeiro R, Machado-Neto JA, Campos Pde M, et al. Ten-eleven-translocation 2 (TET2) is downregulated in myelodysplastic syndromes. Eur J Haematol. 2015; 94:413-418. [PubMed: 25200248]

29. Zhang W, Shao ZH, Fu R, et al. TET2 expression in bone marrow mononuclear cells of patients with myelodysplastic syndromes and its clinical significances. Cancer Biol Med. 2012; 9:34-37. [PubMed: 23691452]

30. Bejar R, Lord A, Stevenson K, et al. TET2 mutations predict response to hypomethylating agents in myelodysplastic syndrome patients. Blood. 2014; 124:2705-2712. [PubMed: 25224413]

31. Malcovati L, Karimi M, Papaemmanuil E, et al. SF3B1 mutation identifies a distinct subset of myelodysplastic syndrome with ring sideroblasts. Blood. 2015; 126:233-241. [PubMed: 25957392] 


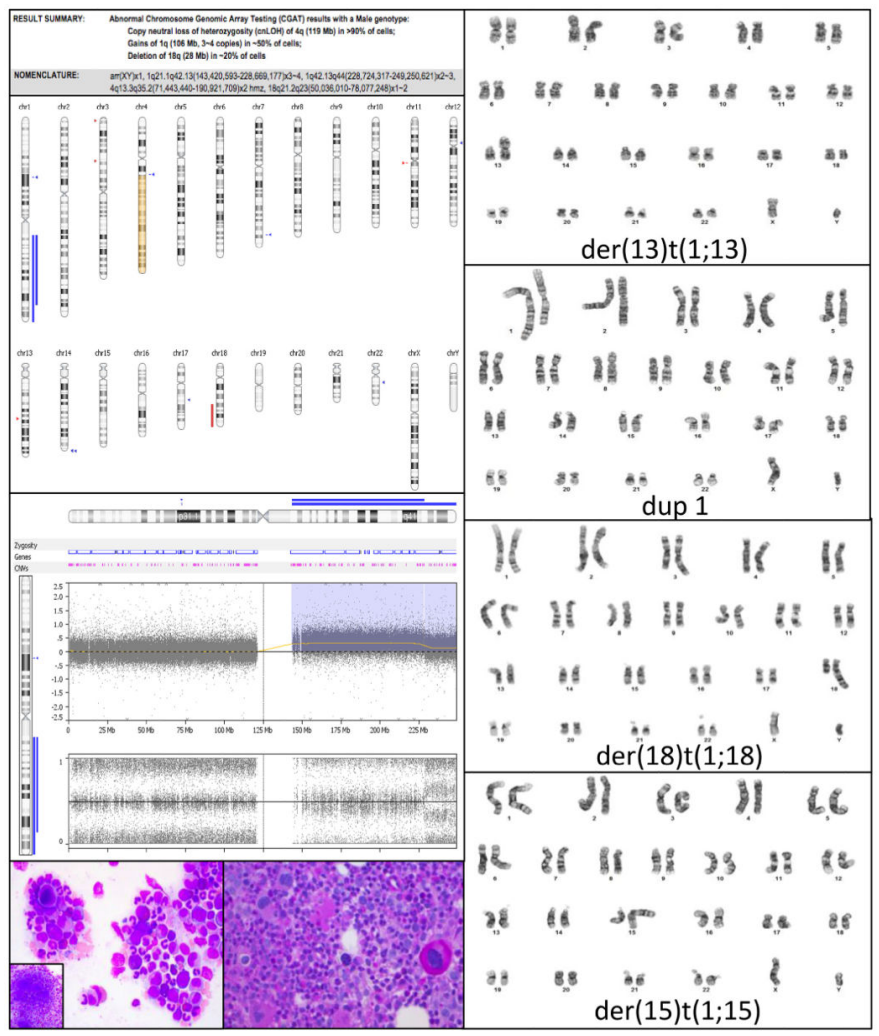

Figure 1.

CGAT results along with the myelodysplastic morphology of the marrow, and the JT karyotype for case 1 prior to HCT. 


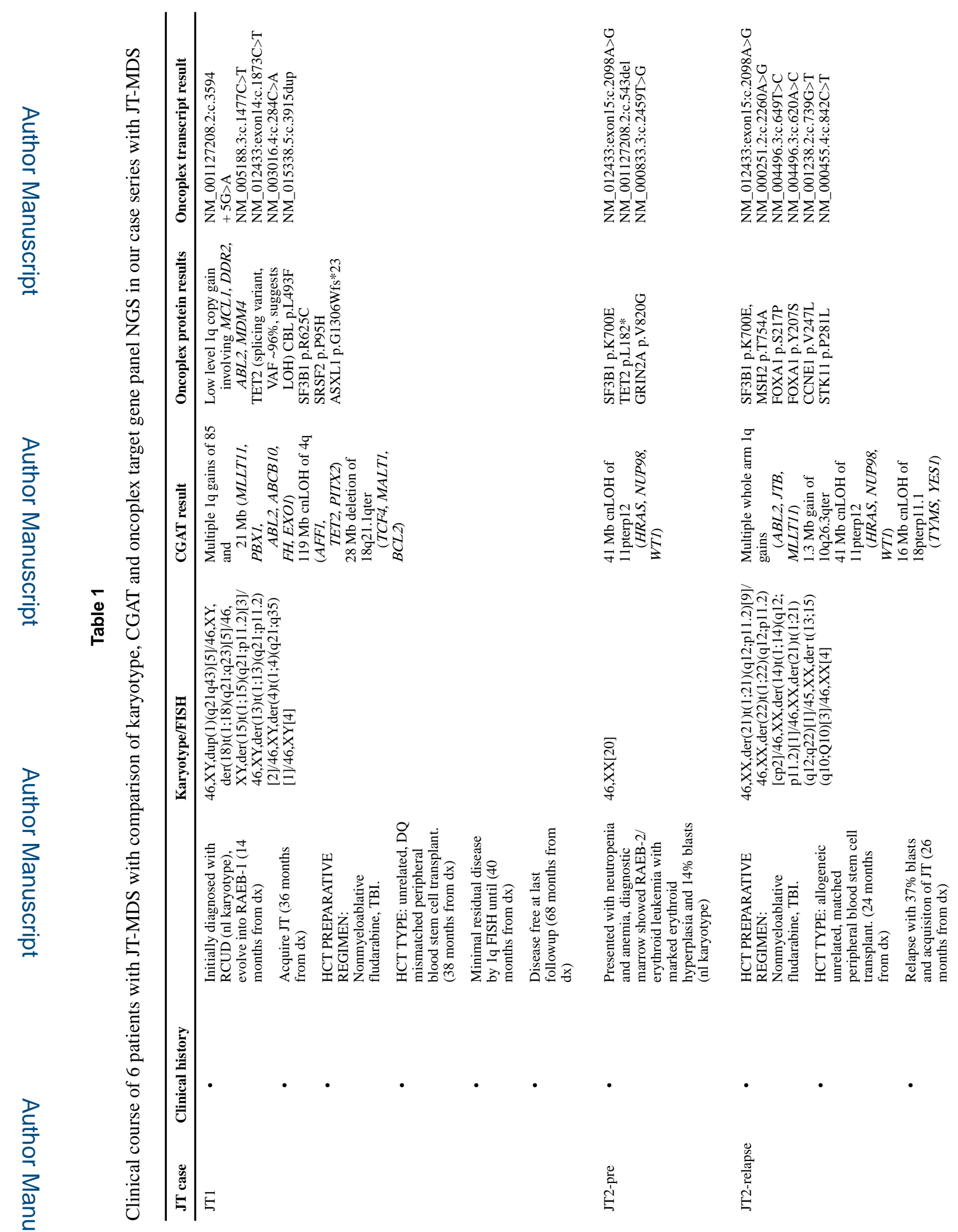

Cancer Genet. Author manuscript; available in PMC 2017 September 01. 
Yeung et al.

Page 11

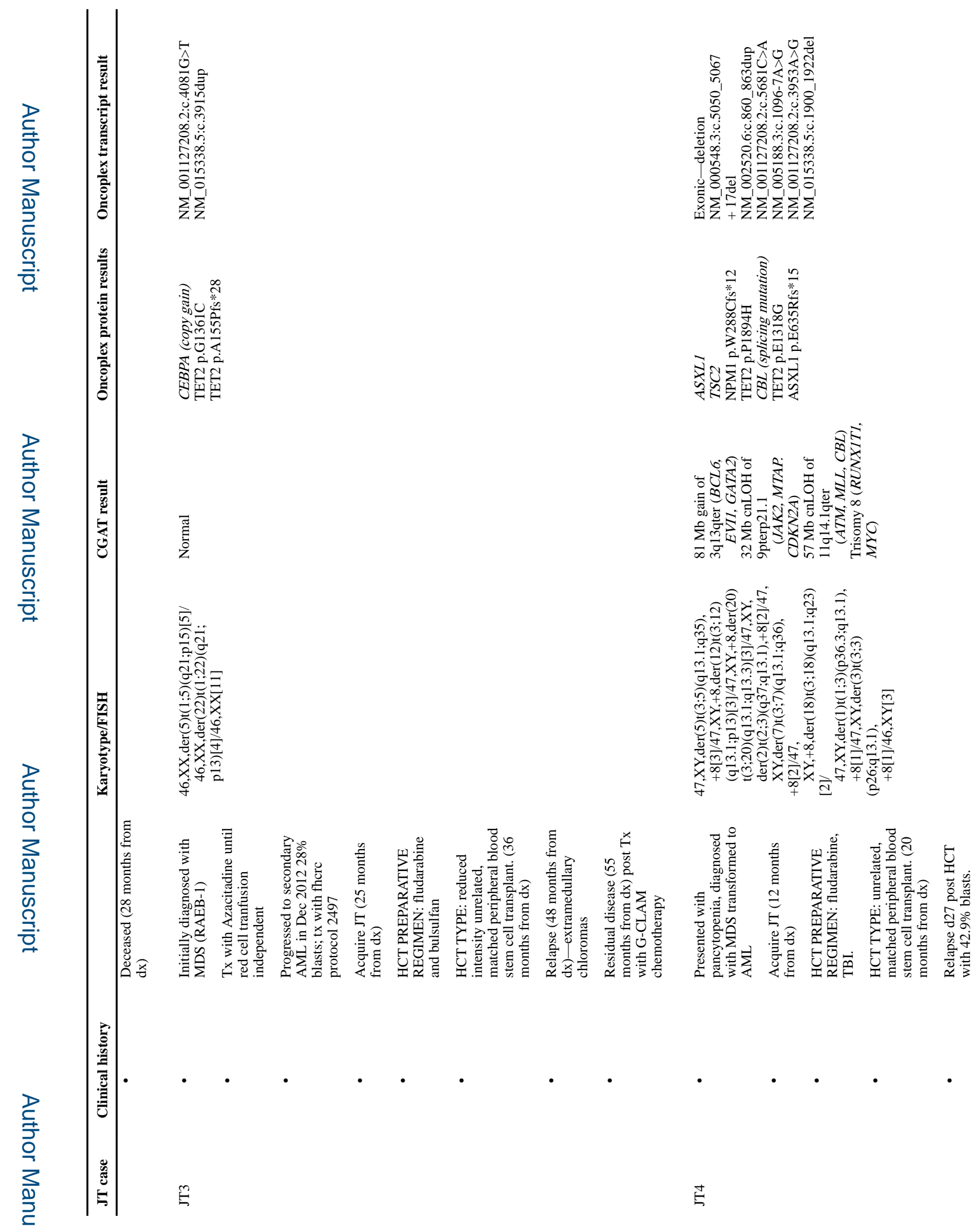

Cancer Genet. Author manuscript; available in PMC 2017 September 01. 


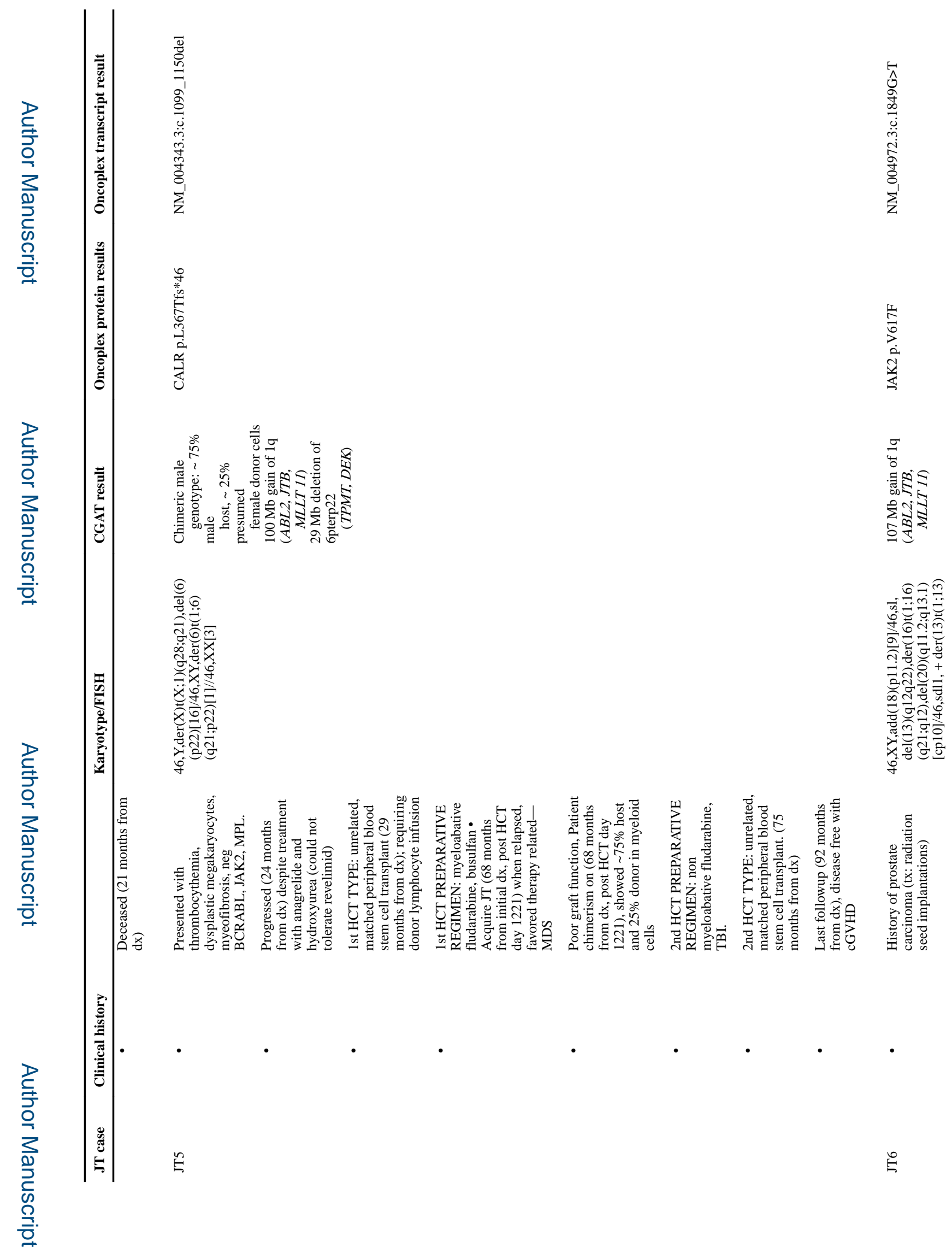


Yeung et al.

Page 13

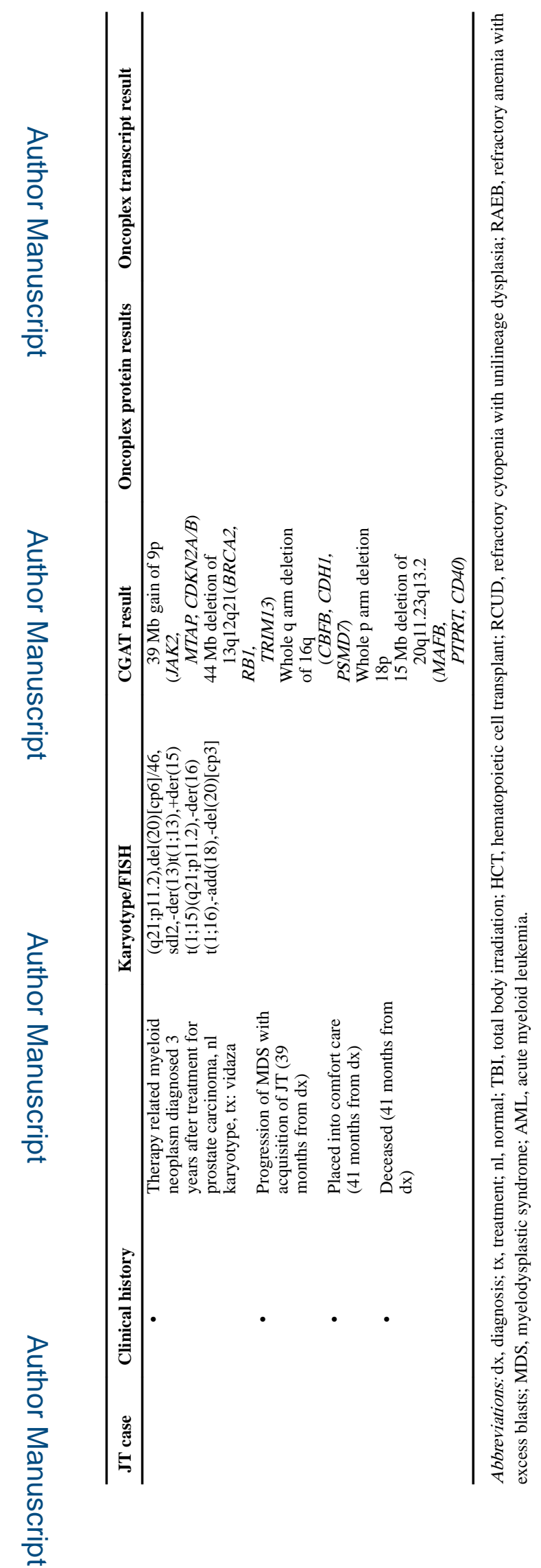

Cancer Genet. Author manuscript; available in PMC 2017 September 01. 
Table 2

Literature review of JT-MDS cases with reported karyotype when available

\begin{tabular}{|c|c|c|c|c|}
\hline Manuscript & Patient age & Diagnosis & Reported clinical course & JT described and karyotype \\
\hline $\begin{array}{r}\text { Hatakeyama } \\
1998(20)\end{array}$ & - & MDS & $\begin{array}{l}\text { Dx with MDS for } 10 \text { years, then } \\
\text { progression to AML M5 }\end{array}$ & $\begin{array}{l}\text { Fusion point at 1q21 46, } \\
\text { XX,der(7)t(1;7)(q21;p22)[10]/46, } \\
\text { XX,der(7)t(1;7)(q21;p22),der(3)t(1;3)(q21;q29)[10]/46, } \\
\text { XX,der(7)t(1;7)(q21;p22),der(4)t(1;4)(q21;p16)[8]/46, } \\
\text { XX,der(7)t(1;7)(q21;p22),der(5)t(1;5)(q21;q35)[34]/46, } \\
\text { XX,der(7)t(1;7)(q21;p22),der(7)t(1;7)(q21;p22)[4]/46, } \\
\text { XX,der(7)t(1;7)(q21;p22),der(8)t(1;8)(q21;q24.3)[10]/ } \\
\text { 46, XX,der(7)t(1;7)(q21;p22),der(9)t(1;9)(q21;q35)[14]/ } \\
\text { 46, XX,der(7)t(1;7)(q21;p22),der(13)t(1;13)(q21; } 34)[4] / \\
\text { 46,XX,der(7)t(1;7)(q21;p22),der(21)t(1;21)(q21;q22)[2]/ } \\
\text { 46,XX,der(7)t(1;7)(q21;p22),der(22)t(1;22)(q21;q13)[2]/ } \\
\text { 46,XX[2] }\end{array}$ \\
\hline $\begin{array}{l}\text { McGrattan } \\
2010(21)\end{array}$ & 86 & RAEB-1 & $\begin{array}{l}\text { Presented with normal } \\
\text { karyotype RAEB-1, } \\
\text { transformed in } 20 \text { months to } \\
\text { AML M5 with JT }\end{array}$ & 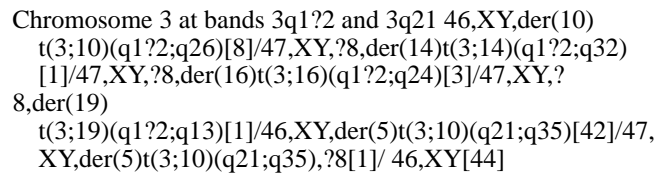 \\
\hline $\begin{array}{l}\text { Lizcova } 2011 \\
\quad(22)\end{array}$ & 1 & RAEB & $\begin{array}{l}\text { Received cord blood transplant } \\
\text { one month after diagnosis } \\
\text { but died on day } 45 \text { from } \\
\text { acute complications (toxicity, } \\
\text { sepsis, and graft versus host } \\
\text { disease) }\end{array}$ & $\begin{array}{l}\text { Chromosome 1q22e1qter 58,XX,pX,pX,p4,p6,p10,der(13) } \\
\text { t(1;13)(q?;q?31), p14,p14,p17,p18,p18,der(19)t(8;19) } \\
\text { (?;q?13.3),p21,p21 [11]/57,XX,pX,pX,p4,p6,p10,der(13) } \\
\text { t(1;13)(q?;q?31),pder (13)t(8;13) (?;q?12),p14,p17,p18, } \\
\text { p21,p21[4]/46,XX[1] }\end{array}$ \\
\hline $\begin{array}{l}\text { Najfeld } 2010 \\
\quad(23)\end{array}$ & - & $\begin{array}{l}\text { MDS } \\
\quad(5 \text { cases })\end{array}$ & $\begin{array}{l}4 \text { of } 5 \text { patients transformed to } \\
\text { AML from MDS, mean time } \\
\text { (range) from JT to AML }=9 \\
(0-42) \text { months, mean time to } \\
\text { development of JT was } 21 \\
\text { months }\end{array}$ & 1q No karyotype data reported \\
\hline $\begin{array}{l}\text { Najfeld } 1995 \\
\quad(24)\end{array}$ & 56 & RAEB & $\begin{array}{l}\text { Transformed to AML and died } \\
1 \text { yr after progression to } \\
\text { RAEB }\end{array}$ & 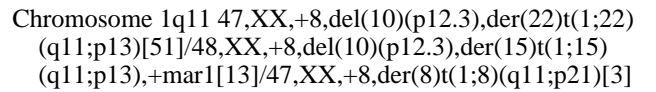 \\
\hline
\end{tabular}

Abbreviations: RAEB, refractory anemia with excess blasts; MDS, myelodysplastic syndrome; AML, acute myeloid leukemia. 